\title{
Research on CAM Based on Mass Customization
}

\author{
Chuxuan Wei \\ Huazhong University of Science and Technology, Wuhan, 430074
}

Keywords: mass, customization, CAM system

\begin{abstract}
The core contents of CAM system read NC code by making use of computer programming and machine tool equipment so as to improve the operating efficiency of machine tool equipment as well as to save the running cost of enterprises. Based on the theory of Mass Customization, this paper will analyze NC instruction generation technology in CAM system, management operation of processing information, automatic processing, database integration, information exchange between machine tools, and other key technologies.
\end{abstract}

\section{Introduction}

CAM is also called Computer Aided Manufacturing System. It realizes the application of computer technology in manufacturing and production. The traditional process planning needs not only a lot of manual work, but also is prone to errors. While CAM is able to effectively change this situation. CAM system is programmed for batch processing, usually with the double functions of operation automation and data conversion, which can be widely used for the control of multiple devices.

\section{Key Technologies of CAM System Based on Mass Customization}

Mass customization not only needs to quickly respond to customer requirements to design products, but also is required to achieve the production of mass customization products on the basis of design. In mass customization environment, the function attributes of CAM system which can quickly respond to customer requirements have been updated and programmed from machining instructions in a simple CAD environment to be a whole automation process, covering NC instructions production, management and automated transportation functions. CAM system can realize the timely generation of NC program, unified management of processing information and processing process, as well as automatic transmission and reprocessing instructions in DNC mode. CAM system is a rapid solution to manufacturing problems which integrates mass customization technology, management technology of PDM data information, CAD technology, database hormone, Internet technology, DNC technology, numerical control technology and other projects.

For mass customization of CAM system, related enterprises are supposed to be able to meet the actual needs of customers in the process of processing and manufacturing, that is, a rapid generation solution of NC instructions for product variant design in CAD system. In the process of CAD operation based on mass customization platform, property sheet and part master model related technology are used to analyze or extract the characteristic parameters of the part during the design work of corresponding product or part, and property sheet of the part model is constructed to make use of the master model during mass customization to manufacture quickly products that customer wants. In this case, the inherent interactive generation function of CAM system is not suitable for the rapid production and processing requirements of corresponding products, so it is necessary to establish a NC document for the parts, and use it to process the NC program of variant parts. On the basis of quick response to customers' demand, the variant design and product configuration of parts are carried out, and characteristic parameters are to be found from the corresponding parts' property sheet, so as to drive NC documents and produce the NC program system for processing parts in time.

In the process of mass customization, an enterprise is capable of managing all information data 
effectively by using CAM system, including customer and supplier information managed by CRM or SCM system, product structure and document information managed by PDM system, and surplus inventory information managed by ERP system in enterprise and so on. On the basis of using network technology, the above information has become a relatively complete unified system and, in many stages, promotes the realization of mass customization goals of enterprises. In the CAM system based on mass customization, it is required that the information used in parts processing in the production process should be managed uniformly, including the NV program, prop data, parts engineering drawings and other materials used in the process of parts processing, which are required to be managed and organized scientifically and effectively. The whole process acquires to ensure the safe, orderly and unified storage of data information. Doing a good corresponding work can effectively enhance the controllability of enterprises in operation, management, production and processing.

As for the production situation of mass customization in workshop, CAM system also put forward corresponding requirements, containing the historical data of product processing and production process in workshop, as well as the processing and management of these data, so as to know the workshop production situation at any time. The historical data information mainly includes the name of the part that has been processed, the name of NC program and machine tools, quantity, time, operator, and processing details, etc. The management of above information can be baed on the PDM system and database system time limit of enterprises. PDM and database systems contain open data ports, which facilitate the integration of data into CAD, DNC and other systems, and submit as well as acquire quickly the data needed by CAM through the use of data interface in the corresponding system. Only in this way, can the data be effectively organized and circulated rapidly within enterprises.

In the CAM system based on mass customization, the transfer of machining instructions to NC machine tools in an important part, which is mainly operated by DNC system. DNC system in CAM system oriented to mass customization is a key link in expanding DNC system in the common sense from the independent existence of workshop system to the information chain of enterprises, which is an important way for the integration of equipment and information in enterprise information system, thus realizing the information exchange inside and outside workshop. In addition, it also can effectively screen the differences existing in the CNC system of workshop CNC equipment and put forward a unified information interface to the information layer of enterprises. Specifically, the main problems of DNC system in CAM system based on mass customization to be solved can be divided into two aspects. On the one hand, it is the communication between DNC system and CNC machine tool, and the transmission of machining instructions. On the other hand, it is the centralized realization of the processing of parts and production information in PDM (data information base) of enterprises.

In order to ensure that the DNC system can be seamlessly integrated with the existing PDM database of enterprises, so as to realize data conversion and processing information management in the workshop, it is necessary to complete the integration of database in CAM system based on mass customization. Automated product design and data manufacturing in manufacturing enterprises are centrally managed by specific product data management systems, so that information can be smoothly conveyed in the process of design, production and manufacturing. Therefore, it is necessary to integrate the traditional DNC system from the workshop independent information into the enterprise database system, which is conducive to improve the efficiency of information exchange inside and outside the workshop. The integration of DNC system and PDM system requires the dual support of communication network and distributed database. The communication network in enterprises mainly uses Ethernet, and the network communication protocol is the IP protocol widely used in the Internet.

CAM system based on mass customization needs to realize effective information exchange between different types of CNC machine tools. It requires DNC system to communicate effectively with multi-type CNC machine tools in PDM system management process. In order to achieve effective communication between DNC system and CNC machine tools, not only the connection 
and bidirectional communication between DNC system and multiple machine tools of CNC system need to be considered, so as to realize the transmission of processing information and feedback of processing status, but also the convenience of communicating with multi-model CNC systems as well as the future development of enterprises and the needs of system integration, that is, to achieve a scalable and economical workshop DNC solution strategy, is to be taken into account.

Generally, there are three ways to realize the networking between DNC mainframe and the underlying NC system in workshop: serial communication, LAN and fieldbus. Computer LAN and fieldbus technology can realize long-distance connection to field facilities. However, LAN has the drawbacks of complex network protocols, weak real-time and anti-interference, while the underlying CNC facilities also need to have intelligent network interface. Fieldbus technology has its own independent protocol, and needs to use the corresponding development tools and development platform, so it is expensive to use. In addition, it is difficult to achieve linkage operation between different platforms and equipment from different manufacturers, which make it not suitable for DNC transformation in the workshop of small and medium-sized enterprises. Compared with the above two technologies, the serial communication structure is relatively simple, easy to operate and cheap, so it is a more practical solution, which is also more suitable for DNC system of small and medium-sized enterprises. Many key technologies in CAM system based on mass customization are relatively independent unit modules in functionality, but they are also an interdependent and inseparable whole ${ }^{[1]}$.

\section{Definition and Key Technologies of Mass Customization}

The concept of mass customization was first proposed in 1970. It is a production method that makes use of standardized models or mass production costs and time to satisfy customers' specific requirements for products and services. In 1987, this production mode was officially defined as mass production, also known as MC. Although mass customization production has not been generally accepted until now, the core meaning is usually positioned as that "in the acceptable price and acceptance time, through the customization of products to meet their individual needs, to enhance customer satisfaction, and the core competitiveness of enterprises in the economic market”. Enterprises using mass customization are able to not only satisfy customer's personalized customization, but also have the same cost and time as mass production.

Mass customization can integrate enterprise, customer, supplier and environment as a whole. Under the guidance of system thought, it can optimize the whole idea and make the best use of the existing resources in enterprises. Through standardized technology, modern design methods, information technology and advanced manufacturing process, according to the specific requirements of customers, as an advance production method, it can achieve low cost, high efficiency, high quality customized products and services. Reducing the diversity inside the products is prone to increase the diversity outside the products. The diversity inside the product is generally manifested in parts, components, tools, materials, processes, abrasives and so on. While the external diversification of a product mainly refers to the selection of types, different styles, various specifications and so on, which customers need. Mass customization requires the reduction of internal product diversification on the premise of passing product technical standards, thereby weakening its design and manufacturing costs, while at the same time increasing external diversification as far as possible within the cost allowance.

Mass customization mainly proceeds from three aspects: business processing, product architecture and information technology. The background of mass customization is the development and survival of traditional manufacturing enterprises in the current information-based social environment. Mass customization makes full use of the parts and components resources existing in enterprises, thus maximizing the efficiency of product development and design, and on the basis of high product quality, reducing its production and development costs to meet the needs of modern enterprises for time, cost, personalized services and other aspects. The main technologies for mass customization are design technology, standardization technology of product information, manufacturing technology, management technology, information technology, etc. Design 
technology mainly includes the theoretical construction of product design method, product reference model information and high-efficiency product design technology and so on. Product information standardization technology includes product standardization and normalization technology, reorganization of manufacturing systems and processes, modular manufacturing and modular equipment etc. Manufacturing technology contains enterprise resource management, enterprise business process reengineering and process management. Information technology is the most important, including data warehouse technology based on mass customization, system development technology and information system integration technology and so on ${ }^{[2]}$.

In the above key technologies of mass customization, product information standardization is the basis of design manufacture and management for mass customization, and also the precondition of mass customization production, among which, the design and manufacture are the goal and method to realize production. Mass customization management refers to the management of enterprises, as well as the application in the operation of enterprises. As for the other four key technology means, only through the use of modern information technology, especially the Internet and computer technology, can we really realize the mass customization production.

\section{Conclusion}

In summary, CAM based on mass customization can be extended to an integrated process including NC instruction management, generation and automatic transportation. By analyzing its key technologies, the implementation of CAM system in mass production process is solved for enterprises.

\section{References}

[1] Xu Yaoyang. Secondary Exploration of Interactive CAD/CAE/CAM Systems [J]. Electronic Technology and Software Engineering, 2018 (09): 44.

[2] Cui Yongbo. Research and Development of CAD/CAM System with Two-dimensional and Three-dimensional Cams [J]. Innovation and Application of Science and Technology, 2017 (12): 140. 